
allemande

51-2| 2019

Les Humanités environnementales : circulations et renouvellement des savoirs en France et en Allemagne

\title{
Pratiques de la transition agricole - deux exemples allemands
}

Nepthys Zwer

\section{CpenEdition}

\section{Journals}

Édition électronique

URL : https://journals.openedition.org/allemagne/2015

DOI : $10.4000 /$ allemagne.2015

ISSN : 2605-7913

Éditeur

Société d'études allemandes

Édition imprimée

Date de publication : 10 décembre 2019

Pagination : 407-423

ISSN : 0035-0974

Référence électronique

Nepthys Zwer, "Pratiques de la transition agricole - deux exemples allemands », Revue d'Allemagne et des pays de langue allemande [En ligne], 51-2 | 2019, mis en ligne le 02 décembre 2020, consulté le 18 mai 2021. URL : http://journals.openedition.org/allemagne/2015; DOI : https://doi.org/10.4000/ allemagne.2015 


\section{Pratiques de la transition agricole - deux exemples allemands}

- Nepthys Zwer*

"Nous sommes face à un champ de ruines", déclarait la ministre de l'Agriculture allemande Renate Künast en $2001^{(1)}$, soit un an après le scandale de la vache folle, à propos de l'état de l'agriculture industrielle de son pays. Son discours de l'époque semble avoir contribué à mettre en circulation la notion de «transition agricole» ("Agrarwende»). Le chancelier Gerhard Schröder promettait alors de dire «adieu aux usines agricoles». Si la réforme de la politique agricole initiée par les Verts n'a pas vraiment porté ses fruits aujourd'hui, le terme de «transition agricole» est passé dans le langage courant en Allemagne et désigne un changement de paradigme attendu dans l'agriculture qui, idéalement, devrait être écologique et durable.

La raison du succès d'une approche qui relève davantage du discours performatif que d'une réalité tient aussi au fait que, depuis

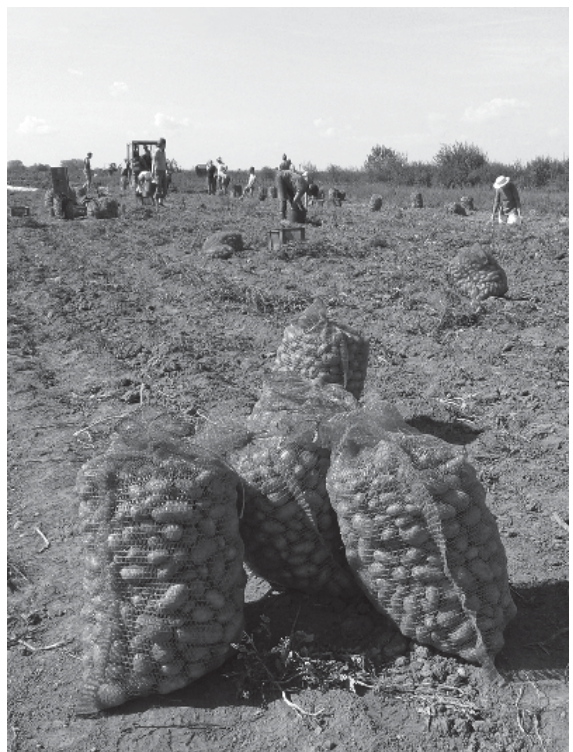

Récolte des pommes de terre, SoLaVie, 15 septembre 2018 (Photo: Nepthys Zwer)

* Docteure en études germaniques, Université de Strasbourg. Membre active de SoLaVie Ortenau e.V. Ce texte est rédigé en écriture inclusive.

1 Renate Künast était alors ministre de la Protection des consommateurs, de l'Alimentation et de l'Agriculture dans le gouvernement de coalition rouge-verte de Gerhard Schröder. Voir les réactions dans Der Kritische Agrarbericht 2006 à propos de sa politique, en ligne: www.kritischer-agrarbericht.de/ fileadmin/Daten-KAB/KAB-2006/Moldenhauer_et_al.pdf. 
vingt ans, des ONG environnementales et le groupe AgrarBündnis ${ }^{(2)}$ mobilisent l'opinion publique et font pression sur le gouvernement. L'idée d'un changement d'orientation nécessaire, voire exigé, du fonctionnement de l'agriculture allemande a depuis été adoptée par nombre de consommatrices et consommateurs.

Le présent article présente deux projets allemands nés de ce désir de changer notre rapport à la terre et à ses productions: l'association de maraîchage solidaire SoLaVie du Bade-Wurtemberg et l'entreprise de commerce alternatif de café Teikei Coffee. Leur point commun est que ce sont deux très jeunes entreprises de l'agriculture solidaire nées, pour la première, d'une initiative locale collective, pour la seconde, de la volonté d'un artiste plasticien ayant fait l'expérience de l'agriculture solidaire en Amérique du Sud. Elles ne s'inscrivent dans aucun programme de politique environnementale municipale ou nationale.

Dans un premier temps, je préciserai les concepts de transition agricole et d'agriculture solidaire, notions analysées également dans une perspective transnationale franco-allemande. Je présenterai ensuite brièvement l'activité de ces deux entreprises et exposerai la façon dont elles conçoivent et articulent leur contribution à la transition agricole. Une mise en parallèle des discours propres aux deux entreprises permet de mettre en évidence la similitude des démarches.

Je m’appuie sur le discours porté par ces groupes dans leurs publications écrites, orales et en ligne. Étant membre de SoLaVie, j’ai également tenu compte des échanges conduits dans ce cadre. Mon analyse de la démarche de Teikei Coffee repose pour partie sur mes entretiens avec l'initiateur du projet ${ }^{(3)}$. En ce sens, cette présentation de deux cas de pratiques socio-environnementales de la transition agricole constitue également un témoignage engagé.

\section{Vers une transition agricole}

La notion d'"Agrarwende» n'a pas d'usage établi en français, tout comme de nombreux termes relatifs à l'agroécologie en circulation aujourd'hui. On rencontre parfois le terme de «transition agricole et alimentaire» ${ }^{(4)}$ pour lequel une recherche en ligne montre qu'il est souvent le fait d'institutions et organismes associatifs, alors qu'en Allemagne, on le trouve aussi sur les sites des actrices et acteurs de terrain. Une requête dans le moteur de recherche Google renvoie dix fois plus d'occurrences pour des ouvrages en allemand consacrés au sujet (6630 pour le terme «Agrarwende», 677 pour le terme «transition agricole»). Dans le champ de la recherche, le Guide des Humanités environnementales, paru en 2016 et qui

2 L'AgrarBündnis réunit 25 organisations (issues de l'agriculture, de la protection de l'environnement, de la défense des consommateurs, etc.) et compte plus d'un million de membres, www.agrarbuendnis.de/.

3 Les citations sont placées entre guillemets. Les traductions sont miennes. Du 16 au 18 octobre 2018, j'ai participé avec Hermann Pohlman, le fondateur de Teikei Coffee, aux rencontres d'automne du réseau national allemand des Solawis à Kassel.

4 Pour cette raison, j'ai choisi de traduire le mot «Wende», qui suppose un retournement, par celui de «transition» qui indique plutôt une évolution. En France, on trouve plus souvent la notion de «transition énergétique et écologique», telle qu’employée par le Conseil national de la transition écologique (CNTE) et le Conseil national du développement durable (CNDD). 
dresse l'état des savoirs des Lettres et Sciences humaines et sociales sur la nature, ne recense pas encore ce terme ${ }^{(5)}$.

Il est aussi révélateur qu'il n'existe, à ce jour, pas d'entrée «transition agricole» dans l'encyclopédie en ligne Wikipédia, alors que l'entrée "Agrarwende» a été créée en décembre 2013 déjà. La définition qui y est donnée du terme s'est d'ailleurs maintenue depuis: il s'agit de forcer (forcieren) un changement de paradigme dans la politique agricole par une écologisation (Ökologisierung) de l'agriculture. En Allemagne, le terme est donc entré dans l'usage courant et il détient une réelle charge politique. La manifestation pour une transformation de l'agriculture qui a réuni plus de 30000 personnes à Berlin le 19 janvier 2019 avait pour slogan «Wir haben Agrarindustrie satt!» («Nous en avons marre de l'industrie agricole!») ${ }^{(6)}$. L'exigence de la mise en place d'une agriculture durable ${ }^{(7)}$ est ici indissociable de l'idée d'un refus de l'économie agricole basée sur le profit.

En revanche, la notion d' «agroécologie», en tant que pratique et mouvement, revêt dans les deux langues et les deux pays la même idée d'une application de techniques écologiques à une agriculture étendue à son environnement économique et social. Pour ses défenseuses et défenseurs les plus radicaux, elle prend la dimension d'une «éthique de vie ${ }^{\left({ }^{(8)}\right.}$ tant elle leur semble indissociable de l'écologisme ${ }^{(9)}$. Les représentations de la nature, les grands thèmes écologiques, tels ceux de la crise environnementale ou de l'anthropocène, sont communs aux discours des activistes des deux pays. La transposition des préoccupations agroécologiques dans le domaine des politiques agricoles se heurte par contre aux réalités nationales.

Pour sa réforme annoncée de 2021, la Politique agricole commune (PAC) - dont les subventions conditionnent largement le devenir de l'agriculture européenne - prévoit de laisser à l'entière discrétion des États leurs propres plans d'action stratégiques et les laisse fixer eux-mêmes la priorité des critères (dont les critères environnementaux) conditionnant l'attribution de subventions ${ }^{(10)}$. Or la transposition de préoccupations

5 Aurélie Choné, Isabelle Hajek et Philippe Hamman (dir.), Guide des Humanités environnementales, Villeneuve d'Ascq, Presses universitaires du Septentrion, 2016. Voir notamment l'étude de Josiane Stoessel, «Sociologie de l'agriculture», p. 253-263, qui évoque pour la France un "mouvement local de transformation de l'agriculture» et un «changement de paradigme», p. 260.

6 www.tagesschau.de/multimedia/video/video-495927.html.

7 Sur les usages des notions de «Nachhaltigkeit/ durabilité», voir la contribution de Philippe Hamman, "Quels savoirs de la “durabilité” en France et en Allemagne?» dans le présent dossier.

8 Voir Pierre Rabi, Vers la sobriété heureuse, Arles, Actes Sud, 2010, p. 144: «L'agroécologie que nous préconisons comme éthique de vie et technique agricole permet aux populations de regagner leur autonomie, leur sécurité et leur salubrité alimentaires, tout en régénérant et préservant leurs patrimoines nourriciers.»

9 L'écologisme étant compris comme un engagement actif, réfléchi et moral pour la protection et la préservation de l'environnement et de ses ressources.

10 À ce jour, $80 \%$ des subventions de la PAC reviennent à $20 \%$ des exploitations car elles sont calculées en fonction de leur superficie. La PAC continue de favoriser la concentration économique et les productions exportatrices. Malgré toutes les déclarations d'intention, l'écologie reste le parent pauvre de cette politique. Voir Ulrich JAsper et Christian ReHmer, «Die Zukunft der Direktzahlungen. Über die neuen Pläne der EU-Kommission und die mögliche Vorreiterrolle Berlins ", in: Agrarbündnis e.V., Der kritische Agrarbericht 2018, Ab Bauernblatt, 2018, p. 34-38. Sur les ajustements souhaitables en termes d'agroécologie de la réforme de la PAC, voir Friedhelm STODIECK, «Europa muss wählen - bei 
écologiques dans les politiques publiques nationales se heurte aux conflits d'intérêts des différents agrosystèmes ${ }^{(11)}$, ce qui laisse présager un nouveau statu quo en matière de protection de l'environnement. Les politiques agricoles européennes restent donc largement en deçà des préconisations et programmes d'action des instances mondiales ${ }^{(12)}$.

Le concept d'agroécologie est né dans les pays en voie de développement, les grands perdants de l'industrialisation de l'agriculture et de l'économie de marché mondiale. Ceci explique que la notion de « développement durable» («nachhaltige Entwicklung»), telle qu'introduite par le rapport Brundtland de la Commission mondiale sur l'environnement en $1987^{(13)}$, soit une préoccupation pour le devenir de la planète. L'agriculture, étendue aux questions des modes de consommation, de la préservation des ressources et de l'environnement et du développement social, est l'un des piliers de la gestion écologique souhaitée pour la protection des milieux naturels et le bien-être des sociétés humaines. Cette idée prend le contre-pied de l'image négative d'un monde (forcément) globalisé et converge avec les représentations des mouvements altermondialistes: la mondialisation, pour peu qu'on l'organise de manière juste, serait une chance pour l'humanité.

L'idée du "penser global, agir local» ("global denken/lokal handeln»), telle que promue par l'Agenda 21 (le plan d'action pour le XXI siècle des Nations unies) adopté au sommet de Rio de 1992, souligne l'importance du rôle que jouent dans le développement mondial les actrices et acteurs à leur échelle propre.

Travailler la terre et s'en nourrir pose donc aujourd'hui la question du dysfonctionnement de l'économie agro-alimentaire et de la responsabilité de toutes et de tous. L'agriculture ne peut plus être pensée comme cloisonnée, réduite à un secteur précis de l'économie ou d'un territoire national. Le combat écologiste, porté depuis ses origines par la société civile, entre autres en tant que consommatrice des productions agricoles, s'est en partie vu relayé par les instances nationales et internationales qui reconnaissent de fait son rôle d'interlocutrice politique dans ce domaine ${ }^{(14)}$. Aujourd'hui, l'appel à une réorientation des politiques agricoles semble légitime, revendication exacerbée

Landwirtschaft und Klima», in: AgrarbüNDnis e.V., Der kritische Agrarbericht 2019, Ab Bauernblatt, 2019, p. 25-39.

11 La politique de préservation de la biodiversité ( Nationale Strategie zur biologischen Vielfalt»), décidée par l'Allemagne en 2007, a par exemple permis la renaturalisation de seulement $2 \%$ des forêts allemandes en 2018 (contre les $5 \%$ escomptés pour 2020). Voir László MARÁz, «Wie steht es um den Waldnaturschutz? Zehn Jahre Nationale Strategie zur biologischen Vielfalt», in : AGRARBündnIS e.V., Der kritische Agrarbericht 2018 (note 10), p. 228-232. À titre d'exemple, on peut citer les difficultés rencontrées dans la mise en œuvre des programmes de réintroduction des grands prédateurs dans leur habitat naturel. Voir à ce propos la contribution de Guillaume CHRISTEN, «De la gestion à la naturalité: le lynx vient-il déplacer les savoirs de la nature?» dans le présent dossier.

12 Sur les retombées du Grenelle de l'environnement en France, voir Daniel Boy, Matthieu Brugidou, Charlotte Halpern et Pierre Lascoumes, Le Grenelle de l'environnement. Acteurs, discours, effets, Paris, Armand Colin, 2012.

13 www.diplomatie.gouv.fr/sites/odyssee-developpement-durable/files/5/rapport_brundtland.pdf. Il existe de nombreuses traductions du terme anglais «sustainable», dont «soutenable» en français et «zukunftsfähig» en allemand.

14 Au niveau international, on peut citer l'organisation de paysannes et paysans La Via Campesina, créée en 1993, qui défend le principe de la souveraineté alimentaire mondiale et a signé un accord de coopération avec la FAO en 2013. 
par l'inefficacité constatée des instances politiques, prises dans leurs contradictions, l'accélération des dégradations environnementales et du dérèglement climatique, avec leurs coûts économiques et humains associés.

\section{Qu'est-ce qu'une Solawi?}

L'agriculture solidaire (solidarische Landwirtschaft) est l'une des expressions concrètes de cette préoccupation de l'agroécologie et se veut l'une des actrices de la transition agricole. Elle s'est développée au regard de trois injonctions :

- l'agriculture doit intégrer l'écologisme,

- la planète entière doit assumer sa responsabilité pour la sécurité alimentaire des générations futures,

- la multiplication et la concertation des actions locales seront le meilleur levier de la transition agricole.

Les initiatives spécifiques d'agriculture solidaire sont regroupées sous le terme générique anglais de «Community Supported Agriculture (CSA)» («agriculture soutenue par la communauté», "gemeinschaftsgetragene Landwirtschaft») ${ }^{(15)}$, terme qui ne s'est cependant pas imposé dans les pays non anglophones. Chaque pays utilise ses propres dénominations, ce qui reflète la dynamique bottom-up (allant du bas vers le haut) de ce mouvement: Gemeinschaftsgestützte Landwirtschaft (Gelawi) en Autriche, Gruppi di Acquisto Solidale (GAS) en Italie, Cooperativa Unitaria ou Cooperativa Agroecológica en Espagne, etc. Le nombre de créations de CSA croît aujourd'hui de façon exponentielle.

L'histoire veut que l'idée d'une agriculture solidaire ait vu le jour au Japon au début des années 1970 quand, face aux scandales réitérés de l'industrie agro-alimentaire, des mères de famille ont décidé de s'approvisionner directement auprès de petit.e.s productrices et producteurs de l'agriculture biologique, créant les premières associations «Teikei». En japonais Teikei (tri’kzi:) signifie "coopération». Apparu en Suisse en 1978 avec les Jardins de Cocagne, le concept passa par les États-Unis avant d'être adopté

Créations de Solawis entre 1988 et 2019

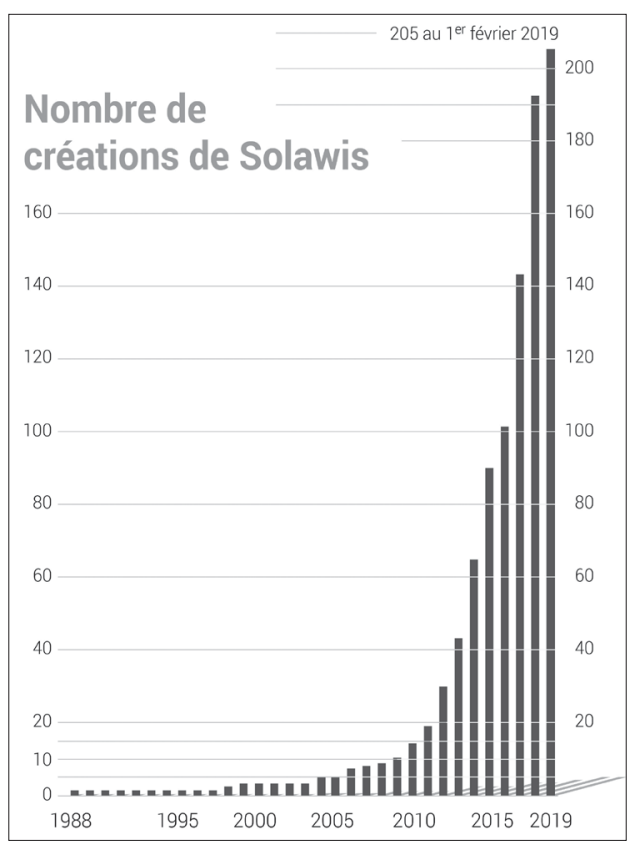

Source: Philippe Rekacewicz, 2019

15 Voir ce rapport sur l'état des CSA: European CSA Research Group, Overview of Community Supported Agriculture in Europe, 2016, en ligne: www.agronauten.net/wp-content/uploads/2016/05/ Overview-of-Community-Supported-Agriculture-in-Europe-final.pdf; le site d'Urgenci, réseau mondial des CSA : urgenci.net/the-network/; Phillip Bitau et al., Solidarische Landwirtschaft - eine soziale Innovation? Eine empirische Studie aus soziologischer Perspektive, 2013, en ligne: www.fb03. uni-frankfurt.de/48975490/Abschlussbericht_SoLawi_final.pdf. 
en Allemagne à la fin des années 1980 (notons qu'en 1968 déjà, le Buschberghof à Fuhlenhagen/Schleswig Holstein appliquait les principes de l'agriculture solidaire). En 2019, on compte 205 Solawis déclarées en Allemagne ${ }^{(16)}$. En France, la première Amap (Association pour le maintien d'une agriculture paysanne), le Jardin des Olivades, a vu le jour à Ollioules/Var en 2001. En 2015, on estime à plus de 2000 les Amap dans le pays ${ }^{(17)}$.

Les Solawis (Solidarische Landwirtschaft - le terme désigne autant la pratique que les fermes elles-mêmes) allemandes ressemblent aux Amap (Associations pour le maintien d'une agriculture paysanne) françaises en ce sens qu'elles permettent à de petites fermes d'assurer leur activité dans un secteur agricole où prévaut la concentration économique. On trouve cependant plus fréquemment en France des fermes simplement fournisseuses de «paniers bio». La démarche davantage politique des initiatives allemandes apparaît dans leur autodéfinition. Les Solawis mettent en avant la nécessité d'une restructuration radicale du secteur:

«Qu'est-ce que l'agriculture solidaire? C'est financer l'agriculture - et non chaque aliment pris séparément. En agriculture solidaire, plusieurs foyers financent une entreprise agricole et reçoivent en échange une partie de la récolte. Les productrices et producteurs entretiennent une relation personnelle avec les consommatrices et consommateurs et découvrent ainsi les avantages d'une agriculture non industrielle et indépendante du marché » ${ }^{(18)}$.

Les AMAP françaises cherchent plutôt à assurer la survie des petites fermes:

«Une AMAP est une Association pour le Maintien d'une Agriculture Paysanne ayant pour objectif de préserver l'existence et la continuité des fermes de proximité dans une logique d'agriculture durable, c'est-à-dire une agriculture paysanne, socialement équitable et écologiquement saine» ${ }^{(19)}$.

Le principe commun est la passation d'un contrat entre une ferme et un groupe de consommatrices et consommateurs qui préfinancent l'activité de production agricole pour une ou deux années en échange de la livraison hebdomadaire de produits généralement issus de la culture biologique. Il s'agit, pour des personnes le plus souvent citadines, de soutenir l'agriculture paysanne tout en s'alimentant de façon saine.

\section{Planter des choux}

SoLaVie e.V. ${ }^{(20)}$, l'association de maraîchage solidaire sise à Neuried-Altenheim, dans la région de l'Ortenau, est une initiative de consommatrices et consommateurs de légumes biologiques. L’association a été créée en 2014 et le projet concrétisé en

16 Chiffres donnés par le réseau allemand des Solawis, créé en 2011: www.solidarische-landwirtschaft. org/. Un film, réalisé par Sylvain Darou et Luciano Ibarra en 2013, présente le concept de la Solawi à l'exemple de la Gartencoop de Fribourg-en-Brisgau: Die Strategie der krummen Gurke, en ligne: www. cinerebelde.org/die-strategie-der-krummen-gurken-p-121.html?language=de.

17 Chiffres donnés par le réseau français Miramap, créé en 2010: miramap.org/-Les-AMAP-.html.

18 Définition proposée par le site Solidarische Landwirtschaft: www.solidarische-landwirtschaft.org/ index.php?id=92.

19 Définition proposée par le site Miramap: miramap.org/-Les-AMAP-.html.

20 Voir le site de l'association: solavie.de/. SoLaVie est l'acronyme de «Solidarisch landwirtschaften und (Leben=)vie» («Cultiver et vivre solidairement»). La consonance française de «c'est la vie» répond à la volonté d'étendre, à terme, l'activité à la France voisine. 
mars 2016. Il approvisionne aujourd'hui 120 familles. Les membres sont dénommé.e.s "personnes prenant une part de la récolte» («Ernteanteilabnehmer.innen»).

SoLaVie produit une cinquantaine de variétés de légumes biologiques non certifiés (la labellisation, avec ses contraintes de contrôle, n'est justifiée que pour une activité commerciale de vente, ce qui n'est pas le cas ici) sur 2,6 hectares de terres en fermage ${ }^{(21)}$. L'association dispose de deux tunnels-serres et loue un hangar où procéder à la répartition des légumes pour ses huit points de distribution. Une partie des récoltes est mise en réserve dans ses propres silos-couloirs.

Le montant de la participation financière des membres est fixé annuellement lors d'une séance d' "enchères" en fonction des capacités de chacun.e et de façon à couvrir le budget prévisionnel. L'association s'est fixée pour principe de rétribuer ses salarié.e.s de façon décente. Elle emploie ainsi, pour l’année 2019, cinq personnes aux champs et à la distribution, dont une personne à temps plein en CDI. Les contrats sont annualisés afin d'absorber les variations saisonnières d'activité.

SoLaVie est une Solawi particulière, car elle n'est ni partenaire d'une ferme, ni ne dispose de sa propre exploitation agricole, ce qui la dénue de l'esprit «entrepreneurial» qui pourrait animer la personne dont dépend la marche d'une entité économique ${ }^{(22)}$. Un groupe relativement stable, le "cercle des actives et actifs» (Aktivenkreis) d'une trentaine de personnes administre et gère l'association sur la base du bénévolat. Les tâches sont réparties entre différents groupes de travail et de projets ad hoc (Arbeitsund Projektgruppen) ${ }^{(23)}$. Les résultats et préconisations de ces groupes sont présentés au cercle des actives et actifs pour délibération et prise de décision.

En raison de l'investissement en temps très important pour les membres actifs, certains préfèrent ne participer qu'aux travaux agricoles ou à la distribution. Les mardis et mercredis matins, jours de récolte, il n'est pas rare de voir une dizaine de personnes s'activer aux champs. Les nombreuses activités réunissent aussi les membres au moment des grandes récoltes, de la fabrication de la choucroute, des fêtes des solstices, feux de camps, nuitées ou de sorties diverses, contribuant à forger un esprit de communauté et une identité propre. Cette convivialité revêt une importance toute particulière pour l'association.

\section{Transporter du café à la voile}

L'organisation à but non lucratif Teikei Coffee opère à un niveau international. Créée en 2017 par l'artiste plasticien Hermann Pohlmann, l'activité fonctionne selon les principes de l'agriculture solidaire, ce que le groupe met en avant en vantant son

21 Les parcelles sont cultivées en bio depuis une vingtaine d'années et les produits respectent certainement les normes biologiques les plus exigeantes. L'association vient de décider de l'éventuel achat d'un terrain, ce qui permettrait de soustraire une parcelle agricole à la spéculation foncière.

22 Sur les différentes formes juridiques et organisationnelles des Solawis, voir: Veikko Heintz, Solidarische Landwirtschaft. Betriebsgründung, Rechtsformen und Organisationsstrukturen, Netzwerk Solidarische Landwirtschaft, 2018.

23 On compte, autour de l'équipe en charge de la culture et de la distribution ainsi que du cercle (auquel est joint un bureau pour les actes juridiques), six groupes de travail fixes: culture, distribution, relations publiques, éducation, communauté, droits et finances. S’ajoutent des groupes formés ponctuellement pour des projets spécifiques comme un recrutement. 
«community supported coffee» ${ }^{(24)}$. Un contrat lie les personnes productrices aux consommatrices et consommateurs, mais la centrale, sise à Fribourg-en-Brisgau, sert d'intermédiaire et prend en charge la gestion administrative de l'activité.

Trois fermes produisant du café au Mexique voient ainsi l'écoulement de leur production annuelle garanti. Le montant total convenu leur est versé à l'avance pour la saison, même en cas de défaut de production. La première année, le préfinancement a été réalisé sur la base des premières commandes et d'une opération de financement participatif. $27 \%$ du prix de vente revient aux productrices et producteurs, soit 7,8 euros par kilo (contre 4,90 euros dans le réseau fairtrade en 2017). Les fermes sont ainsi en mesure de mettre en place des objectifs stratégiques et de procéder à des investissements qu'elles n'auraient pas envisagés sans cette sécurité financière et si leurs bénéfices avaient été tributaires des aléas des prix de vente du café sur le marché mondial. Ces personnes, "expertes de leur métier» et «exigeantes», plantent et récoltent à la main l'arabica dans leurs fincas sur les hauts plateaux de la région de Coatepec, dans l'État de Veracruz, au centre du pays: El Equimite, une ferme qui pratique la complantation (agroforesterie) en préservant $70 \%$ de la forêt et la permaculture, et deux fermes, Los Carilles et Sol nocturno, disposant de moins de 5 hectares de terres agricoles. La concertation entre les fermes, la logistique et l'acheminement vers la côte sont assurés par l'entreprise de transformation et de commercialisation Ensambles, qui cherche à promouvoir la production et la distribution de café et de fruits biologiques du Mexique ${ }^{(25)}$. Elle est une émanation de l'association Bios Comunidad Sustentable, avec laquelle Teikei est en train de développer un réseau de coopération associatif. D’autres petites entités ont ainsi été créées autour du projet (école, centre de recherche

Fonctionnement de Teikei Coffee

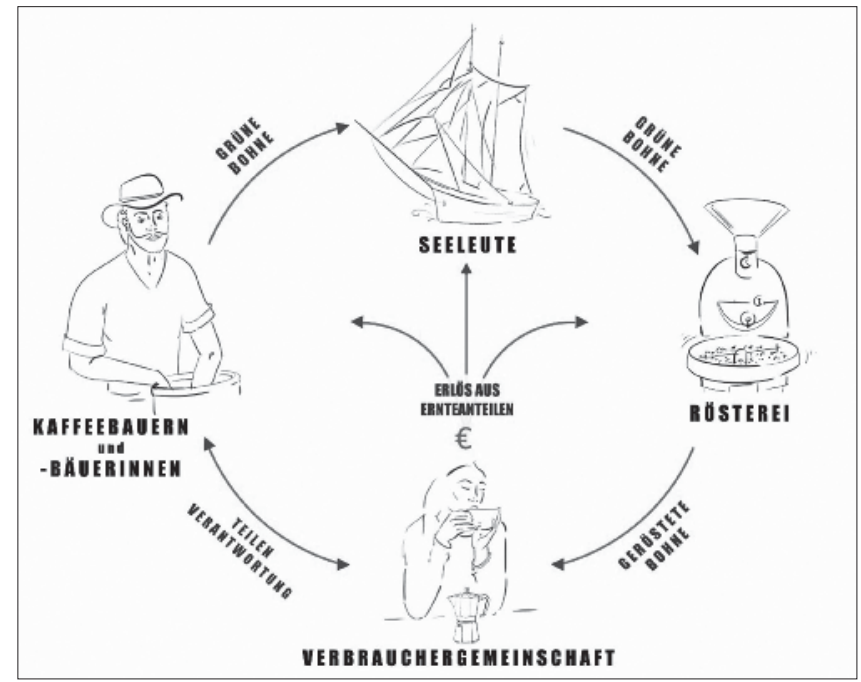

shop.teikeicoffee.org/ueber-teikei

24 Herman Pohlmann a auparavant travaillé avec des CSA brésiliennes (voir note 42 ci-après).

25 www.ensamblescafe.com/. Cette organisation accompagne les fermes pour l'obtention d'une certification biologique ou biodynamique. 
agronomique). L'entreprise Teikei réinvestit entièrement son bénéfice dans ces projets locaux et la formation des paysan.ne.s.

La particularité du projet est son mode de transport du café. La cargaison chargée au Mexique traverse chaque année l'Atlantique à bord d'un voilier, ce service étant assuré par une société maritime pratiquant le «transport maritime durable» ${ }^{(26)}$. Ce transport, qui dure 68 jours, permet de «ménager les écosystèmes maritimes» en raison de l'absence de pollution sonore (Lärmverschmutzung) des mers et du non recours à des énergies fossiles, ce qui permet une réduction de $90 \%$ des émissions des gaz à effet de serre par rapport à un transport conventionnel. Ce mode de transport original assure une identité de marque forte au groupe, le logo de ce «café transporté à la voile» ("gesegelter Kaffee») étant, évidemment, un petit voilier.

Le café est torréfié chaque semaine près de Hambourg et à Bâle, chez de petits torréfacteurs certifiés bio, en fonction des quantités commandées. Il s'agit d'un café arabica en grain haut de gamme qui coûte 27 euros le kilo à partir d'une commande de $10 \mathrm{~kg}$.

La clientèle a donc la certitude de consommer un produit à faible empreinte écologique et issu d'une agriculture plus solidaire. En Allemagne et en Suisse, les acheteuses et acheteurs se regroupent en collectifs d'achat et préfinancent une quantité fixe à leur livrer pour l'année, selon la fréquence souhaitée. En 2017, au lieu des 500 kg escomptés, Teikei a vendu $2500 \mathrm{~kg}$ de café et $4000 \mathrm{~kg}$ en 2018.

Outre son mode de transport et de distribution alternatifs, ce système encourage des pratiques agricoles écologiques et paysannes dans un pays émergent subissant, comme la plupart des pays d'Amérique latine, les effets dévastateurs d'une agriculture industrielle dédiée à l'exportation ${ }^{(27)}$. Sans disposer du label fairtrade, il se réfère à ses principes (dialogue, transparence, respect et agriculture biologique).

En tant que distributeur, Teikei est tributaire de son image et de la qualité de sa communication. Le traitement de l'information sur son site est professionnel et seul le tutoiement à l'adresse des lectrices et lecteurs ( $\mathrm{Du}$ möchtest Teil dieser Gemeinschaft werden?») et le choix de photos d'individus rappellent la nature solidaire de la démarche. L'esthétique visuelle minimaliste, avec très peu d'informations, évoque par association l'idée de simplicité et la transparence.

\section{Comment faire la transition agricole soi-même ${ }^{(28)}$}

On retrouve chez ces deux actrices de l'agriculture solidaire les principes de la transition agricole, à savoir l'écologisme et la conscience de sa propre responsabilité dans le cours des choses.

26 «Nachhaltige Transportkette von der Produktion bis zum Kunden», voir le site de la société: timbercoast.com/en/cargo/.

27 Les effets délétères de l'agrobusiness dans ces pays sont notamment dénoncés par un remarquable travail de cartographie critique: Kollektiv Orangotango+ (éd.), This is not an Atlas, Bielefeld, transcript Verlag, 2018.

28 «Agrarwende selber machen!» est l'une des formules du réseau Solawi. 


\section{Vivre de la nature}

SoLaVie se considère comme faisant partie d'une «indispensable transition agricole» («Wir sehen uns als Teil einer notwendigen Agrarwende»). Teikei a pris le nom de la première initiative d'agriculture solidaire et cherche à transposer le concept de CSA à l'économie associative et, ce, à un niveau mondial ${ }^{(29)}$.

L'agriculture solidaire repose sur l'idée que les êtres humains peuvent vivre en symbiose avec la nature à condition d'en accepter les règles. En effet, partager le risque d'une mauvaise récolte sans en faire porter les conséquences aux seul.e.s productrices et producteurs désactive non seulement les mécanismes de l'économie monétaire et de l'économie de marché, mais implique aussi une attitude humble face à la nature et ses aléas. C'est assumer l'incertitude de la récolte en raison des conditions climatiques et accepter la moindre efficacité des traitements biologiques ou des techniques manuelles. C'est accepter également un moindre rendement et laisser faire la nature.

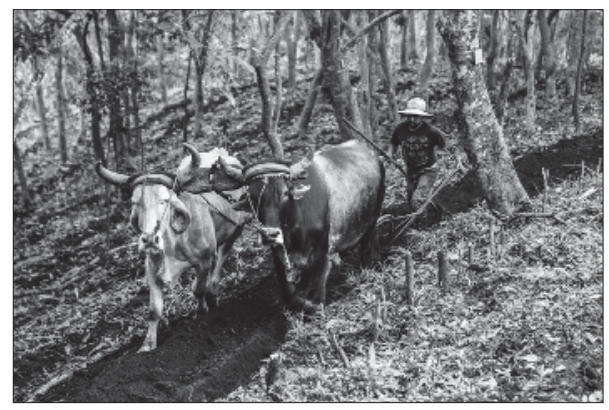

Teikei, labour à la ferme El Equimite

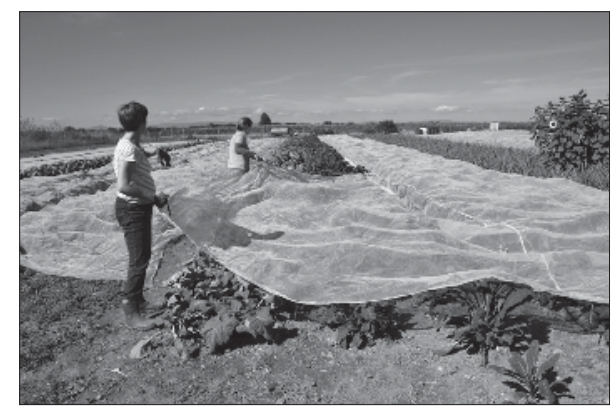

SoLaVie, protection contre les pucerons du chou

Chez Teikei, on « respecte la terre», on veut «travailler avec la nature, plutôt qu'à son encontre». Esteban, de la ferme El Equimite, veut préserver la forêt tropicale, mais en adaptant les techniques modernes à son écosystème. Sa ferme, labellisée Demeter, pratique la culture biodynamique. Si l'agriculture en biodynamie repose sur les principes anthroposophiques de Rudolf Steiner, elle est plutôt mise en œuvre, ici comme ailleurs, pour ses techniques écologiques. Prenant en compte «la réalité sociale, écologique et financière de la région", l'Institut bios terra implanté sur place se conçoit comme un centre d'expérimentation et de transfert technologique qui cherche à «transformer écologiquement» mille hectares de forêt en terre arable. L'organisation partenaire de Teikei Bios comunidad sustentable ${ }^{(30)}$ explique que dans la région de Vera Cruz, grande productrice historique de café, le recours à la chimie a détérioré les écosystèmes et les conditions de vie, "provoquant une crise sociale, culturelle et économique». Mais ces «conflits sociaux, économiques et politiques et ces problèmes environnementaux» sont compris comme «des leçons, des opportunités» qui donnent de «nouvelles impulsions » à la région.

29 Herman Pohlmann: "Ich habe Teikei gegründet mit der Frage, läßt sich die Idee der solidarischen Landwirtschaft auch global denken?».

30 L'organisation propose des modèles commerciaux alternatifs pour l'agriculture dans les domaines de la production alimentaire, la médecine naturelle, la formation, la construction verte, l'écotourisme et la conservation du paysage. 
Les quinze portraits ${ }^{(31)}$ que j'ai réalisés de mes camarades solavistes en 2018 en leur demandant d'expliquer pourquoi elles et ils se considéraient comme «solavistes» montrent que ce respect de la nature («une nature intacte», "l'harmonie avec la nature», «le respect de la terre») est important à leurs yeux.

Pourtant chez SoLaVie, la question écologique n'est pas débattue ni thématisée tant elle semble aller de soi. Il est aussi admis que la nécessaire régénérescence écologique ne pourra se faire que par la main de l'humain ${ }^{(32)}$. Les principes de l'association montrent son approche technique de la résolution du problème environnemental dans l'agriculture :

- production d'aliments biologiques, régionaux et saisonniers sans recours à des engrais de synthèse, des pesticides et la technique OGM,

- planification concertée entre l'équipe de maraîchage et les membres,

- conditions de travail équitables pour l'équipe de maraîchage,

- soutien de l'économie régionale,

- contribution à la préservation de la biodiversité des espèces et de la fertilité du sol,

- contribution à un paysage diversifié et à la protection des animaux,

- réduction de la destruction des aliments par l'utilisation de légumes ne répondant pas aux normes.

L'usage fait ici de la nature ne vise donc pas l'effacement de l'empreinte humaine, mais l'adaptation la plus respectueuse possible à un environnement qui, parce qu'il doit nourrir la population, ne peut être indemne des altérations anthropogéniques. La distinction humain/nature s'efface en faveur d'un rapport de «partenariat» qui implique la légitimité de l'exploitation de la nature si elle se fait en toute responsabilité( ${ }^{(33)}$.

\section{Assumer sa responsabilité politique}

On trouve dans les commentaires accompagnant les portraits deux autres références récurrentes, la première à la qualité biologique, gustative et authentique des légumes, l'autre à la nature du projet en tant qu' «alternative à une industrie agricole (capitaliste)» basée sur la consommation, le gaspillage et l'exploitation des personnes. Ces deux idées sont étroitement liées.

«Manger bio», ce n'est pas seulement faire quelque chose pour sa propre santé, mais aussi et surtout, prendre conscience des implications environnementales et sociales de ses choix de vie personnels. La simple «Biokiste», le «panier bio ${ }^{(34)}$ de beaucoup

31 Portraits réalisés en août 2018 selon la disponibilité des personnes, en ligne: solavie.de/.

32 On mesure l'évolution du discours écologique dans les documentaires, où le catastrophisme qui prévalait jusque dans les années 2010 - il suffit de penser au film Koyaanisqatsi de Godfrey Reggio (1983) ou à Home de Yann Arthus-Bertrand (2009) - a fait place à un optimisme de l'action pour réaliser la transition écologique et sociale, comme l'illustre le documentaire Demain. Partout dans le monde, des solutions existent de Mélanie Laurent et Cyril Dion (2015).

33 Sur la généalogie conceptuelle européenne de l'écologie politique et la critique sociale inhérente à cette notion, voir Patricia ZANDER, «Écologie politique. Des origines aux développements en France», in: Choné/Hajek/Hamman, Guide des Humanités environnementales (note 5), p. 93-102.

34 L'agriculture biologique, contrairement à l'agroécologie, s'accommode d'une production industrielle. Voir sur ce point Maria Нешвисн, "Agrarökologie als Leitbild für Landwirtschafts- und Lebensmittelpolitik. Eine Begriffserklärung», in: Der kritische Agrarbericht 2018 (note 10), p. 39-44. Sur la conventionnalisation du secteur biologique, voir Thomas Poméon, Ève Fouilleux, Sylvaine 
d'Amap, ne peut rivaliser avec les légumes qu'on a contribué à faire pousser et qu'on a récoltés soi-même, que l'on vient chercher dans le point de distribution hébergé par d'autres solavistes et que l'on pèse soi-même, au gramme près sur un pèse-bébé pour ne pas léser les autres. Selon Carlos, le maraîcher de SoLaVie, «savoir d'où viennent les produits, participer à leur production" est une grande chance et une façon de se réapproprier son mode d'existence. Les «Konsumenten» deviennent des «Prosumenten " (Produzent+Konsument) responsables ${ }^{(35)}$. La pratique de l'agriculture solidaire subvertit ainsi le système actuel de production et de distribution des aliments. La politique salariale de l'association, qui a fixé le salaire horaire à 15 euros bruts, soit un salaire largement supérieur aux usages du secteur, montre bien cette corrélation entre une subversion économique et politique et la plus-value symbolique quacquièrent des aliments produits sans exploitation capitaliste des salarié.e.s.

La prise de responsabilité des activistes de l'agriculture solidaire se traduit dans un mode de production artisanal hautement technique qui permet de traiter la terre avec circonspection: sélection de semences reproductibles, qui, en raison de leur pollinisation naturelle, contribuent à préserver la biodiversité génétique, méthodes biologiques de lutte contre les insectes ravageurs, techniques alternatives de conservation, fabrication de kimchi, de jus de pomme... Tout un savoir hautement spécialisé, une culture se (re)constituent autour de l'activité.

Ce savoir-faire est mis en commun, transmis par les expert.e.s aux membres et par les parents aux enfants. En raison de l'intérêt croissant que soulève le projet, les membres accueillent régulièrement des groupes (des écoles locales, des groupes de recherche, la presse...) sur le terrain, ce qui a poussé l'association à créer le nouveau groupe de travail «Éducation» (AG Bildung). Le projet devient ainsi un véritable modèle de maraîchage alternatif.

Teikei pousse aussi les fermes mexicaines à développer les techniques de culture adaptées à l'environnement de la forêt et à reconstituer un savoir-faire ancestral. Les fermes familiales doivent être en mesure d'assurer la transmission de ce savoir et de l'activité à leurs enfants. Au Mexique, Cecilia, qui dirige l'école locale Bios Lila Montessori, veut créer chez les enfants une conscience de la nature et de l'alimentation saine et leur apprendre comment produire leurs propres aliments et comment vivre en communauté et «sensibiliser le monde à l'avenir».

\section{Identité locale - action globale}

Bien que les deux activités soient différentes dans leur structure, on constate donc une grande similitude dans la manière dont elles abordent leur contribution à la transition agricole. Cette ressemblance s'exprime aussi dans la façon dont elles se conçoivent elles-mêmes en se positionnant en tant que véritables actrices de l'économie et par le sens qu'elles donnent à leur propre communauté d'action.

LEMEILleur et Allison Loconto, «L'agriculture biologique en France, entre projet critique et conventionnalisation", in: Gilles Allaire et Benoit DAVIron, Transformations agricoles et agroalimentaires. Entre écologie et capitalisme, Versailles, Éditions Quae, 2017, p. 181-198. 


\section{Un système fractal}

Le périmètre de l'activité de SoLaVie est restreint à une quinzaine de kilomètres pour le point de distribution le plus éloigné. La production régionale de légumes a bien sûr une raison d'être écologique: des transports réduits évitent la pollution, l'absence d'emballages évite le gaspillage, aucune réfrigération électrique n’est nécessaire, etc. Mais l'association s'assigne aussi sa propre place et se localise géographiquement. On peut comprendre l'importance de cette assignation dans l'espace comme la recherche d'un ancrage symbolique, qui donne au groupe une identité claire et souligne son attachement à la terre. Mais il positionne aussi l'association par rapport aux autres partenaires de la transition et acteurs économiques (Solawis voisines, réseau, organismes et institutions officielles) et établit clairement sa nature d'actrice locale dans le marché agricole mondialisé des multinationales et des global players. SoLaVie reconstitue entièrement un circuit court de production-distribution-consommation qui lui est propre et qui correspond à un circuit économique alternatif en miniature. Selon le principe du "penser global, agir local», la démultiplication des actions locales finira par instaurer de bonnes pratiques et infléchira notre façon de concevoir et pratiquer l'agriculture. La création de réseaux de l'agriculture solidaire il y a une dizaine d'années, tant en France qu'en Allemagne, appuie encore cette dynamique.

L'assignation spatiale est aussi très marquée chez Teikei, dont les pôles d'activité se répartissent entre plusieurs pays. La production au Mexique, la traversée de l'Atlantique, la localisation des équipes administratives et des groupes consommateurs sur une carte donnent l'image d'un globe terrestre presque petit où il est possible de fédérer des équipes très différentes. Les actrices et acteurs conservent leur culture, ce qui est mis en avant par le respect de leur langue, voire de leur accent espagnol ou de la Suisse alémanique. La portée symbolique du transport est évidente: le voilier établit physiquement le lien entre les groupes. Ici, par la fédération des différentes entités, on invente ex nihilo un réseau dans lequel les personnes apprennent à se connaître et coopèrent malgré les distances. Ce mode de fonctionnement est rendu possible par l'utilisation d'Internet, qui se montre, partout, le meilleur allié des initiatives citoyennes ${ }^{(36)}$.

La constitution de ces réseaux permet aux deux entreprises de s'insérer de façon alternative dans le processus économique, étant assurées de partager une démarche commune avec les autres actrices et acteurs qui œuvrent activement à la transition agricole, écologique et socio-économique, et de décider ainsi de la «forme de la globalisation ${ }^{(37)}$ :

«Nous sommes au XXI ${ }^{\mathrm{e}}$ siècle - nous vivons dans un monde qui devient systémique, s'accélère et s'optimise. Cela représente des chances et des libertés, mais aussi des risques et des dégâts. Qui en profite? Qui en paie les frais? Qu'advient-il des gens à l'autre bout du monde, que se passe-t-il avec la nature, quelles conséquences cela a-t-il pour les générations suivantes? Nous vivons dans un monde plein de contradictions. Nous sommes à la fois proches et éloignés les uns des autres, reliés et pourtant étrangers, nous produisons et

36 La plateforme de commande en ligne de produits régionaux La Ruche qui dit oui! (idée reprise sous le terme de food assembly en Allemagne), créée en 2011, illustre parfaitement les nouvelles opportunités offertes par le numérique dans le domaine de l'agroécologie. Voir en ligne: laruchequiditoui.fr/fr.

37 Voir les textes de la rubrique «Schwerpunkt: Globalisierung gestalten» dans Der Kritische Agrarbericht 2018 (note 10). 
détruisons en même temps. Avec Teikei, nous prenons nos responsabilités et articulons efficacement entre eux de bons concepts » ${ }^{(38)}$.

Ce réseau a une architecture fractale, où chaque entité fédère derrière elle d'autres participant.e.s et s'insère elle-même dans un mouvement d'opérations jumelles plus vaste. Gibran, de Bios comunidad sustentable, se voit à la tête d'« un projet intégral de développement humain au travers d'entreprises qui créent un bien commun qui respecte à la fois la nature, les cultures locales et les gens».

\section{Communauté et démocratie participative}

Se mettre en réseau, c'est avant tout créer du lien social. Parmi les éléments saillants du discours des deux initiatives, on remarque combien prévaut la notion de «communauté» (Gemeinschaft). Il s'agit là d'un moteur particulièrement puissant dans ces démarches.

Autant il semble aller de soi chez SoLaVie, qui est l'œuvre d'un collectif, autant il est étonnant chez Teikei. Et pourtant il est l'argument mis en avant sur son site: la «communauté» est formée par les paysans, les marins, les torréfacteurs et la communauté des consommateurs, sans oublier le «Teikei Team» en charge de l'organisation. Les agricultrices et agriculteurs partenaires du Mexique sont présenté.e.s avec leurs photos et un film, titré Comment on peut vivre en communauté, les montre à l'œuvre et explique leur démarche. Les acheteuses et acheteurs sont invité.e.s à se regrouper pour leurs achats et appelé.e.s à devenir une part de la communauté ( Hier kannst du nun Teil der Teikei Gemeinschaft und der Teikei Bewegung werden!»). Teikei cherche à créer entre les partenaires «un rapport d'égal à égal», et ce en dépit des distances et des différences de culture, car cette communauté se veut ouverte et respectueuse des altérités.

Chez SoLaVie, le respect réciproque se manifeste notamment dans les réunions du cercle, où chaque avis est pris en compte. De longs débats permettent de clarifier la plupart des questions, quitte à y revenir lors de séances ultérieures. L'association met en œuvre des outils de gouvernance de type sociocratique, notamment la recherche du consentement pour les décisions importantes (prises, le cas échéant, à la majorité des trois quarts) ${ }^{(39)}$. La longue tradition de la vie associative en Allemagne et de l'engagement citoyen dans la vie de la cité jouent certainement pour beaucoup dans le succès de ce mode de fonctionnement ${ }^{(40)}$.

Pour ses membres, l'association est un véritable «terrain d’apprentissage» ("Verein als Lernfeld») pour apprendre à fonctionner dans un groupe et à faire vivre ce groupe sur la base de l'intelligence collective. SoLaVie expérimente ainsi le fonctionnement de la démocratie participative et de la gestion collective de la production d'un bien, d'une économie «resocialisée», réappropriée par la société civile. Créer une communauté d'action, c'est inventer une nouvelle forme de démocratie.

38 teikeicoffee.org/kaffeebauern/.

39 Sur la notion de sociocratie et les règles de l'auto-organisation, voir en ligne: les-jours-heureux.fr/ wp-content/uploads/2016/10/La_Sociocratie_Buck_Edenburg_Charest.pdf.

40 Voir Nepthys ZwER, «Une resocialisation du maraîchage», visionscarto.net (2018), en ligne: visionscarto.net/socialisation; version allemande: visionscarto.net/SoLaVie-Gemuseanbau-alsSozialisierungsexperiment. 
À cet égard, l'exemple de Teikei peut apporter des éléments de réponse pour comprendre ce qui motive ces démarches d'une réappropriation de l'économie par la société civile. Son fondateur veut «montrer qu'une façon associative de pratiquer l'économie a une incidence positive sur toutes les personnes qui y participent». En tant qu'artiste plasticien disciple de Joseph Beuys ${ }^{(41)}$, il pense que la créativité de chaque personne peut servir à pratiquer un «art social» qui, en réalisant collectivement une "plastique sociale», modifiera la structure sociale, aussi bien sur le plan politique qu'économique ${ }^{(42)}$. Cette conception d'une société malléable et modifiable par les individus qui la constituent permet tout simplement une réappropriation de la chose publique par une société civile qui s'auto-organise. L'accompagnement par les instances publiques de ces initiatives privées dites «transformatrices» («transformative Unternehmungen») indique que celles-ci sont appelées à jouer un rôle croissant dans une reconsidération du fonctionnement du secteur agro-alimentaire ${ }^{(43)}$.

\section{Conclusion}

Dans le cadre restreint de cet article, il n'est pas possible d'explorer plus avant les différents aspects de cet engagement citoyen pour la transition agricole. Il faudrait, par exemple, se pencher sur les rapports que l'agriculture solidaire entretient avec la tradition et les valeurs qu'elle promeut. Ainsi, à l'ancrage historique de Teikei dans la tradition centenaire de la solidarité agricole répond chez SoLaVie le recours aux méthodes et techniques artisanales. Ces choix impliquent une reconsidération de notre rapport au temps: travailler aux champs, naviguer à la voile, c'est accepter et apprendre à apprécier le temps lent.

Les pistes à explorer, autant dans le champ de l'histoire des idées que des études sociologiques des pratiques quotidiennes, sont innombrables: la généalogie conceptuelle de cette écologie politique, les canaux de transmission des savoirs sur la nature et l'environnement, l'articulation entre les concepts de solidarité et d'entraide et celui de responsabilité, l'importance accordée à la notion de communauté, la construction de l'identité de ces groupes, les modes de mise en place de la démocratie participative, le processus de fédération avec les autres initiatives de l'agriculture solidaire, le rapport entre travail salarié et bénévolat, etc.

Si ces initiatives de l'agriculture solidaire s'avèrent des objets d'études passionnants, c'est en raison de leur nature expérimentale et innovante, mais aussi en raison de la rapidité avec laquelle ces pratiques se répandent aujourd'hui sur toute la planète.

41 Le sculpteur et performateur Joseph Beuys (1921-1986) a forgé le concept de «plastique sociale» (aussi «sculpture sociale») qui restitue leur dimension politique aux entreprises humaines. Voir Jan Ulrich Hasecke, Soziale Plastik. Die Kunst der Allmende. Ein Essay zum 30. Todestag von Joseph Beuys, ebook, 2016.

42 Hermann Pohlmann a expérimenté cette démarche auprès de l'Associação Comunitária Monte Azul, créée par Ute Craemer dans une favela de São Paulo. Cette institution à vocation sociale fonctionne selon les principes de l'anthroposophie et de la «plastique sociale».

43 «Wie können transformative Wirtschaftsformen, statt in der Nische zu verharren, Innovationsprozesse in Gang setzen?", se demande-t-on sur le site de nascent, projet de recherche du ministère de l’Éducation et de la Recherche sur les initiatives de production alimentaire alternatives. En ligne: www.nascent-transformativ.de/projekt/ziele/. 
Les Solawis commencent d'ailleurs à porter un regard autoréflexif sur elles-mêmes, comme le prouve la création récente, par l'entremise de leur réseau, d'un groupe de recherche dédié à l'étude de son propre mouvement ${ }^{(44)}$.

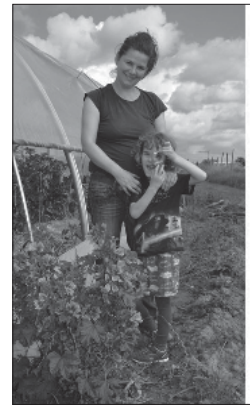

Ich bin Solavistin,

weil ich es toll finde, dass sich Menschen zusammentun und unhabhängig von Politik und Wirtschaft so ein tolles. und wichtiges Projekt auf die Beine stellen.

Außerdem schmeckt das Gemüse super und ich habe tolle und interessante Menschen kennengelernt. Marita
Janis (6 jahre)

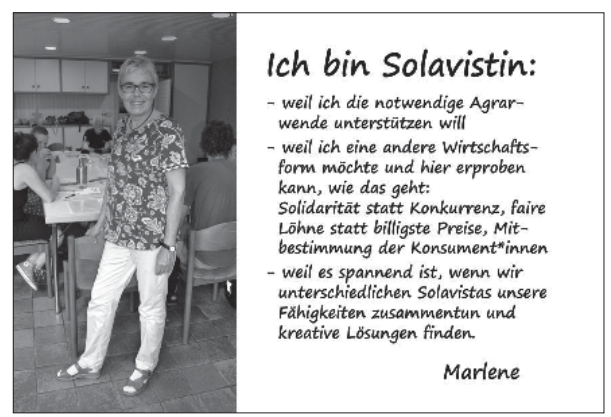

\section{Ich bin Solavistin:}

- weil ich die notwendige Agrarunterstützen will öchte und hier erproben

Solidarität statt Konkurrenz, faire statt billigste Preise, Mitahigkeiten zusammentun und Mariene

Dans le cas de l'agriculture solidaire, la perspective transnationale apporte aux études des humanités environnementales une ouverture dans le champ de l'histoire des idées, notamment politiques ${ }^{(45)}$. L'étude comparée de ce phénomène en France et en Allemagne suppose, par exemple, d'interroger la nature de l'engagement citoyen au regard de l'histoire de la pratique associative et du rapport citoyen au politique dans les deux pays ${ }^{(46)}$. En effet, si la diffusion de l'idée d'une transition agricole semble a priori le fait de la meilleure représentation politique du mouvement écologique en Allemagne, une analyse à la micro-échelle des actrices et acteurs de la société civile révélerait les autres mécanismes à l'œuvre. Comme le montre une simple superposition, le terme français de «transition écologique» suggère un cadre de référence large (l'écologie), alors que le terme d' «Agrarwende/ transition agricole» adopte une focale plus resserrée (l'agriculture), conforme à son caractère opérationnel. De même, bien que les Amap et les Solawis relèvent de la même catégorie de l'agriculture solidaire,

44 www.solidarische-landwirtschaft.org/das-netzwerk/arbeitsgruppen/forschung/.

45 Voir à ce propos les différents programmes de recherche et travaux du CIERA: www.ciera.fr/fr. Voir, notamment, ce travail réalisé dans le cadre du projet de recherche "Saisir l'Europe/Europa als Herausforderung»: Anahita Grisoni et Rosa Sierra (éd.), Nachhaltigkeit und Transition: Politik und Akteure/Transition écologique et durabilité: Politiques et acteurs. Sozio-ökologische Transformation aus deutsch-französischer Perspektive/Regards franco-allemands sur le changement socio-écologique, Francfort-sur-le-Main, Campus, 2018.

46 Voir N. ZwER, «Une resocialisation du maraîchage» (note 40). 
elles ne reposent pas vraiment sur les mêmes postulats (la préservation de l'agriculture paysanne en France, soit une résistance passive et la transformation du fonctionnement de l'économie agricole en Allemagne, soit une résistance active). Sur ce thème précis des perceptions et valeurs qui motivent ces engagements de part et d'autre du Rhin, une étude épistémologique de la circulation et des transferts des idées et des savoirs entre les deux pays s’avérerait particulièrement révélatrice.

Les exemples de SoLaVie et de Teikei présentés ici nous montrent clairement qu'il s'agit de démarches politiques volontaristes et qu'elles doivent être analysées sous l'angle de nouvelles formes d'organisation politique. Ces expériences redessinent clairement le rôle de la société civile en tant quactrice politique qui s'émancipe des tutelles de l'État, des institutions publiques ou des corps intermédiaires. La conscience d'une agrégation, d'une réticulation en cours de ces entreprises alternatives à l'échelle locale et mondiale montre le changement de paradigme qui s'opère aujourd'hui dans la conception du rôle que cette société civile peut et veut jouer dans la polis. Où l'on retrouve l'idée des «multiplicités » interconnectées de Gilles Deleuze et Félix Guattari et où « une branche d'arbre ou une division de racine peuvent se mettre à bourgeonner en rhizome» ${ }^{(47)}$.

\section{Résumé}

L'agriculture solidaire se conçoit comme une alternative à l'agriculture industrielle. En reprenant en main la production de leurs propres aliments, les gens deviennent des acteurs de la transition agricole. À partir des exemples de l'association SoLaVie Ortenau et de l'organisation à but non lucratif Teikei Coffee, le présent article examine comment ce concept peut être mis en cuvre. Chez SoLaVie, les consommatrices et consommateurs financent la production de légumes et reçoivent en retour une partie de la récolte. Chez Teikei Coffee, le café produit dans des fermes familiales au Mexique traverse l'Atlantique en voilier avant d'être distribué aux membres du groupe. L'auteure montre quels rapports ces entreprises entretiennent avec l'écologie et comment elles se conçoivent comme agents responsables et en tant qu'organisations et communautés.

\section{Zusammenfassung}

Die solidarische Landwirtschaft versteht sich als ein Gegenentwurf zur industriellen Landwirtschaft. Menschen übernehmen die Verantwortung für die Herstellung ihrer Lebensmittel und werden somit Akteure der Agrarwende. Der vorliegende Beitrag untersucht an den Beispielen der SoLaVie Ortenau und der nicht-gewinnorientierten Organisation Teikei Coffee, wie sich dieses Konzept umsetzen lässt. Bei SoLaVie finanzieren Verbraucher.innen durch ihre Beiträge die Produktion des Gemüses und erhalten im Gegenzug einen Teil der Ernte. Bei Teikei Coffee wird der von mexikanischen Kaffeebäuerinnen und -bauern nachhaltig erzeugte Kaffee per Segelschiff nach Europa exportiert und an die Teilnehmer.innen verschickt. Es wird gezeigt, wie diese Unternehmen mit dem Thema Ökologie, der eigenen Verantwortung und ihrem Selbstverständnis als Organisation und Gemeinschaft umgehen.

47 Gilles Deleuze et Félix Guattari, Rhizome, Paris, Éditions de Minuit, 1976, p. 44. 